\title{
IL-6R Protective Variant rs7529229 Reduces Interleukin-6 Signaling and Contributes To A Decreased Ischemic Stroke Risk
}

Haihua Zhang

Capital Medical University

Wenbo Zhao

Capital Medical University

Bian Liu

Capital Medical University

Tao Wang

Peking University

Zhifa Han

Tsinghua University

Xunming Ji

Capital Medical University

Guiyou Liu ( $\nabla$ liuguiyou1981@163.com )

Capital Medical University https://orcid.org/0000-0002-1126-2888

\section{Research}

Keywords: ischemic stroke, interleukin-6, genome-wide association study, expression quantitative trait loci analysis, gene expression

Posted Date: October 22nd, 2020

DOI: https://doi.org/10.21203/rs.3.rs-93821/v1

License: (c) (1) This work is licensed under a Creative Commons Attribution 4.0 International License. Read Full License 


\section{Abstract}

Background Interleukin-6 (IL-6) signaling is associated with an increased risk of coronary artery disease (CAD) and ischemic stroke (IS). Growing evidence shows that the minor alleles of IL- 6 receptor gene (IL$6 R$ ) variants rs2228145, rs7529229, and rs4129267 significantly increase soluble IL-6R levels and reduce CAD risk. However, the role of $I L-6 R$ variants in IS is largely unknown, prompting us to perform a comprehensive analysis.

Methods In stage 1 of this study, we performed a meta-analysis of three genome-wide association study datasets from MEGASTROKE, UK Biobank, and the Million Veteran Program to evaluate the association of rs7529229 with IS. In stage 2, we conducted an expression quantitative trait loci analysis to examine the effects of rs7529229 on IL-6R expression in neuropathologically healthy individuals from the UK Brain Expression Consortium, GTEx project, and the eQTLGen Consortium. In stage 3, we used a tissuespecific gene expression analysis to evaluate differences in $I L-6 R$ expression across human tissues using gene expression data from GTEx. In stage 4, we conducted a case-control gene expression analysis to explore the differential expression of $I L-6 R$ in the whole blood of IS patients and healthy controls.

Results We found that: (1) the rs7529229 minor allele significantly reduced the risk of developing IS (odds ratio=0.97, 95\% confidence interval 0.95-0.99, $P=2.30 \mathrm{E}-03$ ); (2) the rs7529229 minor allele significantly reduced $I L-6 R$ expression in relevant tissues especially in blood vessels and whole blood; (3) $I L-6 R$ was mainly expressed in skeletal muscle and whole blood; and (4) IL-6R expression was significantly reduced in the whole blood of healthy controls compared with IS patients. Importantly, the biological senses in stages $1-4$ were all convergent.

Conclusions Taken together, our findings indicate that the rs7529229 minor allele decreases $I L-6 R$ expression in relevant tissues, diminishes IL-6 signaling, and eventually reduces the IS risk. Hence, IL-6R may be a potential therapeutic target for IS. Tocilizumab, a monoclonal antibody that blocks both membrane-bound and circulating IL-6R, might be effective in treating IS or lowering its risk of development, so warrants further testing in suitably powered randomized controlled trials.

\section{Background}

Interleukin-6 (IL-6) is an important inflammatory cytokine that exerts its effects via classic signaling and trans-signaling [1]. In classic signaling, IL-6 binds to the membrane-bound IL-6 receptor (IL-6R) to form an IL-6-IL-6R complex, then recruits glycoprotein 130 (gp130) [2-3]. In trans-signaling, IL-6 binds to soluble IL-6R (sIL-6R), and then binds to membrane-anchored gp130 [2-3]. sIL-6R affects innate and adaptive immune outcomes, and can be considered an important alarmin [4]. IL-6R genetic variants rs2228145, rs7529229, and rs4129267 have been reported to increase sIL-6R levels, which augment the IL-6-sIL-6R complex upon IL-6 binding [4]. Soluble gp130 exclusively inhibits IL-6 trans-signaling without affecting IL6 classic signaling via membrane-bound IL-6R [5]. 
Observational studies have shown that IL-6 signaling is associated with an increased risk of cardiovascular diseases (CVD) including coronary artery disease (CAD) and ischemic stroke (IS) [6-10]. Mendelian randomization (MR) studies have been conducted to evaluate the association between IL- 6 signaling and CVD [11-12]. For example, Rosa et al. applied a reduced IL-6 signaling, increased sIL-6R pattern [11], while Georgakis et al. selected a reduced IL-6 signaling, lower C-reactive protein (CRP) pattern [12]. Interestingly, both MR studies found that increased IL-6 signaling was causally associated with an increased risk of CAD and IS, although inconsistent findings for IS subtypes were reported [11-12]. Hence, the inhibition of IL-6 signaling might be effective in treating CVD or lowering its risk.

Tocilizumab, a monoclonal antibody that blocks both membrane-bound and circulating IL-6R, has been licensed for the treatment of rheumatoid arthritis (RA) to reduce systemic and articular inflammation including CRP and fibrinogen concentrations [9, 13-15]. Tocilizumab binds to the IL- 6 binding site of human IL-6R and competitively inhibits IL-6 signaling [16]. Hence, patients receiving tocilizumab treatment show significantly increased serum IL-6 and sIL-6R levels [16]. Evidence from epidemiological studies indicates that RA patients have a $50 \%$ increased risk of cardiovascular-related morbidity and mortality [17]. Therefore, it is important to clarify whether CVD patients may benefit from treatment with IL-6R blockade using tocilizumab.

Recent studies show that individuals with minor alleles of the $I L-6 R$ genetic variants rs2228145, rs7529229, and rs4129267 not receiving tocilizumab treatment have similar biomarker profiles to those with tocilizumab treatment, as well as a reduced risk of CAD $[9,18]$. In 2012, the IL-6R Genetics Consortium and Emerging Risk Factors Collaboration analyzed 125,222 participants, and found that individuals with the $I L-6 R$ rs 2228145 variant minor allele had $34.3 \%$ increased sIL-6R levels, $14.6 \%$ increased serum IL-6 levels, $7.5 \%$ reduced CRP levels, $1.0 \%$ reduced fibrinogen levels, and a $3.4 \%$ reduced CAD risk [18]. Similarly, the analysis of 133,449 individuals by the Interleukin- 6 Receptor Mendelian Randomisation Analysis (IL6R MR) Consortium in 2012 showed that those with the IL-6R rs7529229 variant minor allele had $9.45 \%$ increased serum IL-6 levels, $8.35 \%$ reduced CRP levels, $0.85 \%$ reduced fibrinogen levels, and a 5\% reduced CAD risk [9]. In 2018, Cai et al. conducted a phenome-wide association study (PheWAS) using MVP datasets of IL-6R variants rs2228145 and rs4129267, and confirmed the significant association of both variants with CAD. The minor allele was shown to reduce the coronary atherosclerosis risk by $5 \%(P=3.43 \mathrm{E}-12)$, the ischemic heart disease risk by $5 \%(P=3.97 \mathrm{E}-$ $12)$, other chronic ischemic heart disease risks by $5 \%(P=6.04 \mathrm{E}-12)$, and the myocardial infarction risk by 6\% $(P=1.69 \mathrm{E}-06)[19]$.

All three IL-6R variants, rs7529229 (chr1: 154448302 [hg38]), rs4129267 (chr1: 154453788 [hg38]), and rs2228145 (chr1: 154454494 [hg38]), are in high linkage disequilibrium with each other [20]. Both rs7529229 and rs4129267 are IL-6R intronic variants, which may regulate the expression of nearby genes [21-26]. The rs2228145 variant is a missense mutation, which replaces a single amino acid residue with another [20]. As well as the involvement of $I L-6 R$ genetic variants in CVD, recent studies also identified rs2228145 as being associated with abdominal aortic aneurysms [27], osteoporotic fracture [28], amyotrophic lateral sclerosis [29], severe heart failure [30], Crohn's disease, and ulcerative colitis [1]. 
rs4129267 was reported to be associated with anxiety [31], asthma [32], and levels of inflammation markers [33].

The association between $I L-6 R$ genetic variant minor alleles and reduced CAD risk is well established and randomized controlled trials (RCTs) have been performed. In 2016, Kleveland et al. conducted a doubleblind, randomized, placebo-controlled phase 2 trial of 117 acute non-ST elevation myocardial infarction (NSTEMI) patients (placebo, $n=59$; tocilizumab, $n=58$ ) [34]. They demonstrated that IL-6 inhibition using tocilizumab attenuated systemic inflammation and troponin T-release in NSTEMI patients, with no significant difference in major safety concerns between the two groups [34]. In 2020, they further found that tocilizumab treatment of NSTEMI patients increased circulating levels of the neutrophil extracellular traps marker citrullinated histone $\mathrm{H} 3$, indicating that tocilizumab could enhance NETosis [35].

While these findings show that human genetic variants can be used to repurpose existing targets for new therapeutic uses $[9,18,36-37]$, the role of $I L-6 R$ genetic variants in IS is largely unknown. In a recent genome-wide association study (GWAS), all three $I L-6 R$ variants, rs2228145 $(P=0.03351)$, rs7529229 ( $P$ $=0.01204)$, and rs4129267 ( $P=0.0264)$, were identified as significantly associated with IS risk, with rs7529229 showing the highest association [38]. Hence, we investigated the rs7529229 variant in our current comprehensive analysis. In stage 1, we evaluated the association of rs7529229 with IS using three GWAS datasets [19, 38-39]. In stage 2, we examined the effects of rs7529229 on IL-6R expression in neuropathologically healthy individuals using three independent expression quantitative trait loci (eQTLs) dataset resources [40-42]. In stage 3, we evaluated potential IL-6R expression differences across different human tissues [43]. In stage 4 , we explored the differential expression of $I L-6 R$ in IS cases and controls using a gene expression dataset [44].

\section{Methods}

\section{Study design}

This study is based on large-scale GWAS summary datasets, eQTLs datasets, expression datasets, and case-control expression datasets. All participants gave their informed consent in the corresponding original studies. All relevant data, analytic methods, and study materials are within the paper. This study does not use animal models.

\section{GWAS datasets}

We selected three different IS GWAS dataset resources. The first is from the largest multi-ancestry metaanalysis of stroke GWAS datasets conducted by MEGASTROKE [38]. Here, we limited our analysis to participants of European ancestry including 34,217 IS cases and 406,111 controls. These include the following numbers of IS subtypes, based on Trial of Org 10172 in Acute Stroke Treatment classification criteria: large artery atherosclerotic stroke (LAS, 4,373 cases and 406,111 controls), cardioembolic stroke (CES, 7,193 cases and 406,111 controls), and small vessel stroke (SVS, 5,386 cases and 406,111 controls) [38]. The MEGASTROKE IS GWAS dataset is publicly available from 
http://www.megastroke.org/index.html. The second IS GWAS dataset resource is from UK Biobank and is publicly available from PheWeb (http://pheweb.sph.umich.edu/SAIGE-UKB/). It includes 1,501 IS cases with cerebral artery occlusion and 399,017 controls [39]. The third IS GWAS dataset resource is from the Million Veteran Program (MVP), and includes 1,198 IS cases with cerebral artery occlusion and 331,601 controls [19].

As a comparison we selected large-scale GWAS datasets for RA and CAD. The RA GWAS dataset is from a large-scale meta-analysis of individuals with European ancestry including 14,361 RA cases and 43,923 controls [45], which is publicly available from http://plaza.umin.ac.jp/ yokada/datasource/software.htm. The CAD GWAS dataset is from the CARDIoGRAMplusC4D consortium ((Coronary ARtery DIsease Genome wide Replication and Metaanalysis (CARDloGRAM) and The Coronary Artery Disease (C4D) Genetics) including 60,801 CAD cases and 123,504 controls, most of European ancestry [46]. It is publicly available from http://www.cardiogramplusc4d.org/data-downloads/. C4D GWAS is a meta-analysis of GWAS studies of individuals of European and South Asian descent (PROCARDIS, HPS, PROMIS, and LOLIPOP) involving 15,420 CAD cases and 15,062 controls [47].

\section{eQTLs datasets}

We examined the association between rs7529229 and $I L-6 R$ expression using multiple eQTLs dataset resources. The first eQTLs dataset resource is from the UK Brain Expression Consortium, which is publicly available from the Brain eQTL Almanac (Braineac) database [40]. Gene expression levels were measured using Affymetrix GeneChip Human exon 1.0 ST arrays [40]. Braineac includes 10 eQTLs datasets of 10 brain tissues from 134 neuropathologically healthy individuals of European descent [40].

The second eQTLs resource is from the Genotype-Tissue Expression (GTEx) project (version 8) including 49 tissues (each with $₫ 70$ samples with genotype data), 828 donors, and 15201 samples [43]. Gene expression levels were measured using Illumina TruSeq RNA sequencing and Affymetrix Human Gene 1.1 ST Expression Array (V3; 837 samples) [43].

The third eQTLs resource is from the eQTLGen Consortium [42]. This consortium conducted a large-scale meta-analysis in 31,684 human whole blood samples from 37 cohorts, with the majority of European ancestry [42]. Gene expression levels were profiled by Illumina, Affymetrix U291, Affymetrix HuEx v1.0 ST expression arrays, and RNA-seq [42].

\section{IS case-control gene expression dataset}

To evaluate the differential expression of $I L-6 R$, we performed an IS case-control gene expression analysis in whole blood using a gene expression dataset from the Gene Expression Omnibus (GEO) database (GSE16561). In this dataset, gene expression profiling was measured in the peripheral whole blood of 39 IS patients (17 men and 22 women) and 24 healthy controls ( 10 men and 14 women) using Illumina microarrays [44]. All 63 participants were of European ancestry [44]. 


\section{Genetic association analysisof IL-6R rs7529229}

We first extracted corresponding summary statistics of the rs7529229 variant from three IS GWAS dataset resources including MEGASTROKE, the UK Biobank, and MVP. We then conducted a metaanalysis to evaluate the association between rs7529229 and IS using R Package (meta: General Package for Meta-Analysis). The overall odds ratio (OR) was calculated by the fixed effects model (MantelHaenszel) or random effects model (DerSimonian-Laird), which was determined by the level of heterogeneity among these three resources [48]. We further investigated the association of rs7529229 with IS subtypes (LAS, CES, and SVS), RA, and CAD using corresponding GWAS summary statistics. The statistical significance for the association between rs7529229 and one specific phenotype was a Bonferroni-corrected threshold $0.05 / 6=0.0083$. Original $P$ values between 0.0083 and 0.05 were considered to be suggestive of an association.

\section{eQTLs analysis}

In Braineac, we first downloaded $/ L-6 R$ expression data and genotype data of variants within $1 \mathrm{Mb}$ upstream of the transcription start site and $1 \mathrm{Mb}$ downstream of the transcription end site [40]. We then evaluated the potential association between rs7529229 and IL-6R expression using linear regression analysis under an additive model by adjusting for several critical covariates including the brain bank, gender, and batch effects in Partek's Genomics Suite v6.6 [40].

In GTEx, eQTLs analysis was performed using FastQTL with the following covariates: top five genotyping principal components, a set of covariates identified using the Probabilistic Estimation of Expression Residuals (PEER) method (the number of PEER factors was determined as a function of sample size [N]: 15 factors for $\mathrm{N}<150,30$ factors for $150 \leq \mathrm{N}<250,45$ factors for $250 \leq \mathrm{N}<350$, and 60 factors for $\mathrm{N} \geq 350$ ), sequencing platform (Illumina HiSeq 2000 or HiSeq X), sequencing protocol (PCR-based or PCR-free), and sex [43]. Detailed information for laboratory and analytical methods was provided in the original paper and the GTEx website (https://www.gtexportal.org/home) [43].

In eQTLGen, a data-driven method was used to integrate gene expression data from platforms [42]. For a given single nucleotide polymorphism (SNP), genes within $1 \mathrm{Mb}$ up/downstream were selected according to the central position of the gene [42]. eQTLs analysis was conducted by a Spearman correlation [42].

\section{Gene expression analysis of IL-6R in GTEx}

We conducted gene expression analysis to investigate $I L-6 R$ expression differences in different human tissues using gene expression data in GTEx (version 8). The gene expression level was quantified by transcripts per million (TPM) based on the GENCODE 26 annotation, then collapsed to a single transcript model for each gene using a custom isoform collapsing procedure [43]. Here, we selected the T test or analysis of variance method to evaluate the potential difference of $I L-6 R$ expression in different human tissues. Statistical significance was set at $P<0.05$. 
We performed a differential expression analysis using the NCBI web application GEO2R (http://www.ncbi.nlm.nih.gov/geo/geo2r/) [49] to invoke the Bioconductor R packages to transform and analyze GEO datasets [49]. Evidence has shown the existence of sex differences in IS epidemiology, presentations, and outcomes [50]. Hence, we further conducted a subgroup analysis by sex. We defined $P$ $<0.05$ as the significance level of differential expression of $I L-6 R$ in IS patients and healthy controls.

\section{Results}

\section{Genetic association analysis of IL-6R rs7529229}

We found no significant heterogeneity among the three IS GWAS dataset resources using Cochran's Q test $(P=0.12)$, so the overall OR was calculated using the fixed effects model. We found that the rs7529229 variant $C$ allele was significantly associated with a $3 \%$ reduced IS risk $(\mathrm{OR}=0.97,95 \% \mathrm{Cl} 0.95-0.99, P=$ 2.30E-03). The single point estimate of each study and overall effect size are provided in Fig. 1, a forest plot for the meta-analysis of rs7529229 in MEGASTROKE, UK Biobank, and MVP. This finding is consistent with RA and CAD GWAS datasets, which showed that the rs 7529229 variant $C$ allele reduced the RA risk by $7 \%(\mathrm{OR}=0.93,95 \% \mathrm{Cl} 0.91-0.96, P=1.90 \mathrm{E}-05)$ and the $\mathrm{CAD}$ risk by $4 \%(\mathrm{OR}=0.96,95 \% \mathrm{Cl}$ $0.94-0.98, P=2.50 \mathrm{E}-07$ ) (Table 1). Using GWAS summary datasets from MEGASTROKE, as provided in Table 1, the rs 7529229 variant $C$ allele showed a possible association with reducing the CES risk by $4 \%$ $(P=2.15 \mathrm{E}-02)$, and the SVS risk by $5 \%(P=4.15 \mathrm{E}-02)$, but not with the LAS risk $(P=3.32 \mathrm{E}-01)$.

Table 1

association of rs7529229 minor allele $C$ with IS, RA and CAD

\begin{tabular}{|c|c|c|c|c|c|}
\hline Traits & OR & $95 \% \mathrm{Cl}$ & $P$ value & Case \# & Control \# \\
\hline Ischemic stroke & 0.97 & $0.95-0.99$ & 2.30E-03 & 36,916 & $1,136,729$ \\
\hline Large artery atherosclerotic stroke & 0.98 & $0.93-1.03$ & 3.32E-01 & 4,373 & 406,111 \\
\hline Cardioembolic stroke & 0.96 & $0.92-0.99$ & 2.15E-02 & 7,193 & 406,111 \\
\hline Small vessel stroke & 0.95 & $0.91-1.00$ & 4.15E-02 & 5,386 & 406,111 \\
\hline Rheumatoid arthritis & 0.93 & $0.91-0.96$ & $1.90 \mathrm{E}-05$ & 14,361 & 43,923 \\
\hline Coronary artery disease & 0.96 & $0.94-0.98$ & 2.50E-07 & 60,801 & 123,504 \\
\hline \multicolumn{6}{|l|}{ OR, odds ratio; $\mathrm{Cl}$, confidence interval; } \\
\hline
\end{tabular}

\section{eQTLs analysis of IL-6R rs7529229}

In GTEx (version 8), eQTLs datasets are available for 47 of 49 tissues, excluding the kidney medulla and esophagus gastroesophageal junction. Hence, a total of 58 tissues (10 tissues in Braineac, 47 tissues in GTEx, and whole blood in eQTLGen) were selected. The eQTLs analysis statistical significance is a 
Bonferroni-corrected threshold of $P<0.05 / 58=8.62 \mathrm{E}-04$, and the original $P$ values between 8.62E-04 and 0.05 were considered to be suggestively significant. In human brain tissues, including 10 from Braineac and 13 from GTEx (version 8), we identified no significant association between the rs 7529229 variant $C$ allele and $I L-6 R$ expression, as shown in Table 2. 
Table 2

rs7529229 variant and IL-6R expression in human brain tissues

\begin{tabular}{|c|c|c|c|c|c|c|c|}
\hline Tissues & EA & NEA & Beta & SE & $P$ value & Samples & Dataset \\
\hline Cerebellar cortex & $\mathrm{C}$ & $\mathrm{T}$ & -0.003 & 0.024 & 0.89 & 134 & Braineac \\
\hline Frontal cortex & $\mathrm{C}$ & $\mathrm{T}$ & 0.040 & 0.022 & 0.07 & 134 & Braineac \\
\hline Hippocampus & $\mathrm{C}$ & $\mathrm{T}$ & -0.022 & 0.026 & 0.40 & 134 & Braineac \\
\hline Medulla & $\mathrm{C}$ & $\mathrm{T}$ & -0.007 & 0.030 & 0.81 & 134 & Braineac \\
\hline Occipital cortex & $\mathrm{C}$ & $\mathrm{T}$ & 0.006 & 0.025 & 0.82 & 134 & Braineac \\
\hline Putamen & $\mathrm{C}$ & $\mathrm{T}$ & 0.029 & 0.027 & 0.29 & 134 & Braineac \\
\hline Substantia nigra & C & $\mathrm{T}$ & 0.005 & 0.032 & 0.87 & 134 & Braineac \\
\hline Temporal cortex & C & $\mathrm{T}$ & 0.022 & 0.025 & 0.38 & 134 & Braineac \\
\hline Thalamus & C & $\mathrm{T}$ & 0.050 & 0.029 & 0.09 & 134 & Braineac \\
\hline Intralobular white matter & C & $\mathrm{T}$ & -0.037 & 0.031 & 0.24 & 134 & Braineac \\
\hline Amygdala & $\mathrm{C}$ & $\mathrm{T}$ & 0.021 & 0.055 & 0.7 & 129 & GTEx \\
\hline Anterior cingulate cortex & $\mathrm{C}$ & $\mathrm{T}$ & 0.051 & 0.057 & 0.37 & 147 & GTEx \\
\hline Caudate & $\mathrm{C}$ & $\mathrm{T}$ & -0.014 & 0.042 & 0.74 & 194 & GTEx \\
\hline Cerebellar Hemisphere & $\mathrm{C}$ & $\mathrm{T}$ & -0.028 & 0.061 & 0.65 & 175 & GTEx \\
\hline Cerebellum & $\mathrm{C}$ & $\mathrm{T}$ & -0.052 & 0.053 & 0.32 & 209 & GTEx \\
\hline Cortex & $\mathrm{C}$ & $\mathrm{T}$ & -0.024 & 0.040 & 0.55 & 205 & GTEx \\
\hline Frontal Cortex & $\mathrm{C}$ & $\mathrm{T}$ & 0.054 & 0.045 & 0.22 & 175 & GTEx \\
\hline Hippocampus & C & $\mathrm{T}$ & 0.0045 & 0.051 & 0.93 & 165 & GTEx \\
\hline Hypothalamus & C & $\mathrm{T}$ & 0.013 & 0.050 & 0.8 & 170 & GTEx \\
\hline Nucleus accumbens & $\mathrm{C}$ & $\mathrm{T}$ & -0.038 & 0.042 & 0.37 & 202 & GTEx \\
\hline Putamen & C & $\mathrm{T}$ & -0.023 & 0.051 & 0.65 & 170 & GTEx \\
\hline Spinal cord & $\mathrm{C}$ & $\mathrm{T}$ & -0.032 & 0.071 & 0.66 & 126 & GTEx \\
\hline Substantia nigra & $\mathrm{C}$ & $\mathrm{T}$ & -0.028 & 0.080 & 0.73 & 114 & GTEx \\
\hline \multicolumn{8}{|c|}{$\begin{array}{l}\text { EA, effect allele; NEA, non-effect allele; Beta is the regression coefficient based on the effect allele. } \\
\text { Beta }>0 \text { and Beta }<0 \text { means that this effect allele increase and reduce disease or phenotype, } \\
\text { respectively. The statistical significance for eQTLs analysis is a Bonferroni-corrected threshold of } P< \\
0.05 / 58=8.62 \mathrm{E}-04 \text {. Meanwhile, the original } P \text { values between } 8.77 \mathrm{E}-04 \text { and } 0.05 \text { were considered to } \\
\text { be suggestively significant. }\end{array}$} \\
\hline
\end{tabular}


In GTEx (version 8) human non-brain tissues, we identified a significant association between rs7529229 and $I L-6 R$ expression in IS-relevant tissues or organs, such as the aorta $(P=1.60 \mathrm{E}-05)$, tibial artery $(P=$ 2.80E-14), and whole blood ( $P=8.5 \mathrm{E}-09$ ) (Table 3). Importantly, only the rs7529229 variant $C$ allele could reduce $I L-6 R$ expression in these relevant tissues and organs (beta $<0$; Table 3 ). It also significantly reduced IL-6R expression in the sigmoid colon ( $P=1.00 \mathrm{E}-04)$, transverse colon ( $P=9.80 \mathrm{E}-13)$, esophagus mucosa ( $P=5.60 \mathrm{E}-05)$, esophagus muscularis $(P=1.50 \mathrm{E}-05)$, and small intestine $(P=1.70 \mathrm{E}-05)$, as shown in Table 3. Additionally, we observed a suggestive association between the rs7529229 variant $C$ allele and reduced $I L-6 R$ expression in cultured fibroblasts $(P=2.60 \mathrm{E}-02)$, the atrial appendage $(P=4.60 \mathrm{E}-$ $03)$, tibial nerve $(P=1.60 \mathrm{E}-02)$, stomach $(P=1.40 \mathrm{E}-02)$, testis $(P=3.80 \mathrm{E}-02)$, thyroid $(P=3.50 \mathrm{E}-03)$, and uterus $(P=2.60 \mathrm{E}-03)$, as shown in Table 3 . We further confirmed the significant association of the rs7529229 variant $C$ allele with reduced $I L-6 R$ expression in whole blood using the eQTLGen dataset (Z score $=-6.6162$ and $P=3.68 \mathrm{E}-11)$. 
Table 3

rs7529229 variant and $I L-6 R$ expression in other tissues in GTEx

\begin{tabular}{|c|c|c|c|c|c|c|}
\hline Tissue & EA & NEA & Beta & SE & $P$ value & Samples \\
\hline Adipose - Subcutaneous & C & $\mathrm{T}$ & -0.052 & 0.037 & $1.50 \mathrm{E}-01$ & 581 \\
\hline Adipose - Visceral (Omentum) & C & $\mathrm{T}$ & 0.052 & 0.029 & 6.60E-02 & 469 \\
\hline Adrenal Gland & C & $\mathrm{T}$ & 0.072 & 0.060 & 2.30E-01 & 233 \\
\hline Artery - Aorta & C & $\mathrm{T}$ & -0.13 & 0.030 & $1.60 \mathrm{E}-05$ & 387 \\
\hline Artery - Coronary & C & $\mathrm{T}$ & -0.14 & 0.074 & 5.50E-02 & 213 \\
\hline Artery - Tibial & C & $\mathrm{T}$ & -0.17 & 0.022 & $2.80 \mathrm{E}-14$ & 584 \\
\hline Breast - Mammary Tissue & C & $\mathrm{T}$ & -0.018 & 0.042 & $6.70 \mathrm{E}-01$ & 396 \\
\hline Cells - Cultured fibroblasts & C & $\mathrm{T}$ & -0.05 & 0.023 & 2.60E-02 & 483 \\
\hline Cells - EBV-transformed lymphocytes & C & $\mathrm{T}$ & -0.02 & 0.111 & 8.60E-01 & 147 \\
\hline Colon - Sigmoid & C & $\mathrm{T}$ & -0.26 & 0.067 & $1.00 \mathrm{E}-04$ & 318 \\
\hline Colon - Transverse & C & $\mathrm{T}$ & -0.3 & 0.040 & $9.80 \mathrm{E}-13$ & 368 \\
\hline Esophagus - Mucosa & C & $\mathrm{T}$ & -0.16 & 0.039 & $5.60 E-05$ & 497 \\
\hline Esophagus - Muscularis & C & $\mathrm{T}$ & -0.17 & 0.039 & $1.50 \mathrm{E}-05$ & 465 \\
\hline Heart - Atrial Appendage & C & $\mathrm{T}$ & -0.14 & 0.048 & 4.60E-03 & 372 \\
\hline Heart - Left Ventricle & C & $\mathrm{T}$ & -0.063 & 0.042 & 1.30E-01 & 386 \\
\hline Liver & C & $\mathrm{T}$ & 0.0098 & 0.052 & $8.50 \mathrm{E}-01$ & 208 \\
\hline Lung & C & $\mathrm{T}$ & -0.018 & 0.029 & 5.30E-01 & 515 \\
\hline Minor Salivary Gland & C & $\mathrm{T}$ & -0.076 & 0.048 & $1.20 \mathrm{E}-01$ & 144 \\
\hline Muscle - Skeletal & C & $\mathrm{T}$ & 0.017 & 0.019 & 3.60E-01 & 706 \\
\hline Nerve - Tibial & C & $\mathrm{T}$ & 0.075 & 0.031 & 1.60E-02 & 532 \\
\hline Ovary & C & $\mathrm{T}$ & 0.054 & 0.054 & 3.20E-01 & 167 \\
\hline Pancreas & C & $\mathrm{T}$ & 0.019 & 0.034 & 5.80E-01 & 305 \\
\hline Pituitary & C & $\mathrm{T}$ & -0.024 & 0.040 & 5.50E-01 & 237 \\
\hline Prostate & C & $\mathrm{T}$ & -0.03 & 0.059 & $6.10 \mathrm{E}-01$ & 221 \\
\hline \multicolumn{7}{|c|}{$\begin{array}{l}\text { EA, effect allele; NEA, non-effect allele; Beta is the regression coefficient based on the effect allele. } \\
\text { Beta }>0 \text { and Beta }<0 \text { means that this effect allele increase and reduce disease or phenotype, } \\
\text { respectively. The statistical significance for eQTLs analysis is a Bonferroni-corrected threshold of } P< \\
0.05 / 58=8.62 \mathrm{E}-04 \text {. Meanwhile, the original } P \text { values between } 8.77 \mathrm{E}-04 \text { and } 0.05 \text { were considered to } \\
\text { be suggestively significant. }\end{array}$} \\
\hline
\end{tabular}




\begin{tabular}{|c|c|c|c|c|c|c|}
\hline Tissue & EA & NEA & Beta & SE & $P$ value & Samples \\
\hline Skin - Not Sun Exposed (Suprapubic) & C & $\mathrm{T}$ & -0.048 & 0.030 & $1.20 \mathrm{E}-01$ & 517 \\
\hline Skin - Sun Exposed (Lower leg) & C & $\mathrm{T}$ & -0.048 & 0.032 & 1.30E-01 & 605 \\
\hline Small Intestine - Terminal Ileum & C & $\mathrm{T}$ & -0.24 & 0.053 & $1.70 \mathrm{E}-05$ & 174 \\
\hline Spleen & C & $\mathrm{T}$ & -0.041 & 0.037 & $2.60 \mathrm{E}-01$ & 227 \\
\hline Stomach & C & $\mathrm{T}$ & -0.089 & 0.036 & $1.40 \mathrm{E}-02$ & 324 \\
\hline Testis & C & $\mathrm{T}$ & -0.11 & 0.052 & $3.80 \mathrm{E}-02$ & 322 \\
\hline Thyroid & C & $\mathrm{T}$ & -0.095 & 0.033 & 3.50E-03 & 574 \\
\hline Uterus & C & $\mathrm{T}$ & -0.33 & 0.106 & 2.60E-03 & 129 \\
\hline Vagina & C & $\mathrm{T}$ & -0.067 & 0.084 & 4.20E-01 & 141 \\
\hline Whole Blood & C & $\mathrm{T}$ & -0.084 & 0.014 & $8.50 E-09$ & 670 \\
\hline \multicolumn{7}{|c|}{$\begin{array}{l}\text { EA, effect allele; NEA, non-effect allele; Beta is the regression coefficient based on the effect allele. } \\
\text { Beta }>0 \text { and Beta }<0 \text { means that this effect allele increase and reduce disease or phenotype, } \\
\text { respectively. The statistical significance for eQTLs analysis is a Bonferroni-corrected threshold of } P< \\
0.05 / 58=8.62 \mathrm{E}-04 \text {. Meanwhile, the original } P \text { values between } 8.77 \mathrm{E}-04 \text { and } 0.05 \text { were considered to } \\
\text { be suggestively significant. }\end{array}$} \\
\hline
\end{tabular}

\section{Gene expression analysis of IL-6R in GTEx}

Gene expression analysis showed that $I L-6 R$ is mainly expressed in skeletal muscle (TPM median $=$ 67.24) and whole blood (TPM median = 52.69), with no significant difference in expression between these two tissues. However, $I L-6 R$ expression in skeletal muscle and whole blood was significantly higher than in other tissues $(P<0.05)$ such as the liver (TPM median $=38.03)$, lung (TPM median $=29.45)$, esophagus muscularis (TPM median $=27.04)$, sigmoid colon (TPM median $=25.73)$, esophagus gastroesophageal junction $($ TPM median $=25.38)$, spleen $($ TPM median $=24.00)$, uterus $($ TPM median $=23.29)$, and small intestine (TPM median $=22.03)$.

$I L-6 R$ showed very low expression in human brain tissues. Of all GTEx (version 8 ) human tissues, the 10 lowest $I L-6 R$ expressing were from the brain including the caudate (TPM median $=3.079$ ), hypothalamus (TPM median $=3.037)$, frontal cortex (TPM median $=2.753)$, amygdale (TPM median $=2.737)$, hippocampus (TPM median $=2.709)$, nucleus accumbens (TPM median $=2.571)$, anterior cingulate cortex (TPM median = 2.514), putamen (TPM median = 2.486), cerebellum (TPM median = 1.643), and cerebellar hemisphere (TPM median $=1.185)$. Box plots for $I L-6 R$ expression in different tissues are shown in Fig. 2.

\section{Case-control gene expression analysis}


In human whole blood, we identified significant dysregulation of $I L-6 R$ expression in IS cases compared with controls $(P<0.05$, Table 4). Importantly, we only found significantly increased $I L-6 R$ expression in IS cases (Table 4). For the two IL-6R transcripts, ILMN_1754753 and ILMN_1696394, IS cases showed 37\% $(P=2.33 \mathrm{E}-05)$ and $30 \%(P=7.90 \mathrm{E}-04)$ increased $I L-6 R$ expression compared with controls, respectively. This was further supported by subgroup analysis by sex, as shown in Table 4.

Table 4

IS case-control gene expression analysis of $I L-6 R$ in blood

\begin{tabular}{|lllll|}
\hline Comparison models & ID & Fold change & $P$ value & Dataset \\
\hline IS vs. Controls & ILMN_1754753 & 1.37 & $2.33 \mathrm{E}-05$ & GSE16561 \\
\hline IS vs. Controls & ILMN_1696394 & 1.30 & $7.90 \mathrm{E}-04$ & GSE16561 \\
\hline IS vs. Controls in males & ILMN_1754753 & 1.52 & $8.39 \mathrm{E}-04$ & GSE16561 \\
\hline IS vs. Controls in males & ILMN_1696394 & 1.43 & $2.50 \mathrm{E}-03$ & GSE16561 \\
\hline IS vs. Controls in females & ILMN_1754753 & 1.28 & $5.59 \mathrm{E}-03$ & GSE16561 \\
\hline IS vs. Controls in females & ILMN_1696394 & 1.22 & $3.65 \mathrm{E}-02$ & GSE16561 \\
\hline The significance level is defined to be $P<0.05$. & & & \\
\hline
\end{tabular}

\section{Discussion}

IL-6 signaling has previously been associated with an increased risk of CAD and stroke [6-8]. Hence, inhibiting IL-6 signaling through blockade of the IL-6R may reduce inflammation, such as by treatment with tocilizumab which is licensed for RA therapy [9, 13-15]. Some individuals with the minor allele of the IL-6R rs7529229 variant not receiving tocilizumab treatment show similar biomarker profiles to those receiving tocilizumab treatment $[9,18]$, suggesting that this allele could reduce the risk of CAD, and that CAD patients may benefit from treatment with an IL-6R blockade $[9,18-19]$. However, the role of $I L-6 R$ genetic variants in stroke remains unclear. We performed a comprehensive analysis using large-scale GWAS datasets, eQTLs datasets, gene expression datasets, and IS case-control gene expression datasets and discuss our findings by comprehensive comparisons with previous studies.

In stage 1 of the present study, we found that the IL-6R rs7529229 variant minor allele $C$ significantly reduced IS risk by $3 \%(\mathrm{OR}=0.97,95 \% \mathrm{Cl} 0.95-0.99, P=2.30 \mathrm{E}-03)$ in a meta-analysis of three large-scale GWAS datasets from MEGASTROKE, UK Biobank, and MVP including 36,916 IS patients and 1,136,729 controls. This finding is consistent with previous GWAS in RA and CAD [45-46], as well as with earlier findings from candidate variant analysis and PheWAS analysis $[9,18,19]$.

In stage 2 , we demonstrated that the $I L-6 R$ rs 7529229 variant minor allele $C$ significantly reduced $I L-6 R$ expression in the aorta, tibial artery, and whole blood using large-scale eQTLs datasets from Braineac, GTEx (version 8), and eQTLGen. In 2012, the IL6R Genetics Consortium Emerging Risk Factors 
Collaboration found that the rs2228145 minor allele was not associated with IL-6R mRNA levels or IL-6 production in monocytes [18]. High expression of $I L-6 R$ in relevant tissues or organs is associated with increased IL-6R signaling and CAD risk $[11,51]$. Hence, findings from genetic association analyses and eQTLs analysis are consistent in suggesting that the rs7529229 variant $C$ allele may first reduce $I L-6 R$ expression in relevant tissues or organs, further diminish IL-6 signaling, and eventually lower the risk of $C A D$ and stroke. We consider this an explanation for why individuals with $I L-6 R$ variant minor alleles not receiving tocilizumab treatment have similar biomarker profiles to those with tocilizumab treatment $[9$, 18].

In stage 3, we detected the highest $I L-6 R$ expression in skeletal muscle and whole blood using gene expression data from GTEx (version 8). IL-6R expression in both tissues was significantly higher than in the liver, lung, esophagus muscularis, sigmoid colon, esophagus gastroesophageal junction, spleen, uterus, and small intestine. The lowest $I L-6 R$ expression was found in human brain tissues, which may explain why eQTLs analysis detected no significant association between rs7529229 and IL-6R expression in human brain tissues.

In stage 4, we identified significantly increased IL-6R expression in IS cases compared with healthy controls using whole blood IS case-control gene expression data. High IL-6R expression was previously reported to be associated with increased IL- 6 signaling $[11,51]$. Therefore, our findings are consistent with recent Mendelian randomization studies, which identified IL-6 signaling as causally associated with an increased risk of CAD and IS [9, 11-12]. In stages 1 and 2, we found that the rs7529229 variant $C$ allele lowered the risk of IS, and reduced $I L-6 R$ expression in relevant tissues of healthy individuals, respectively. The observed significantly reduced expression of $I L-6 R$ in healthy controls indicates that the biological senses in stages $1-4$ were convergent.

Our current study had several limitations. First, we limited our analysis to participants of European ancestry including GWAS summary datasets, eQTLs datasets, expression datasets, and case-control expression datasets. However, the association of the rs7529229 variant with IS risk and IL-6R expression may differ among different ancestries. Therefore, our findings should be validated in other ancestries using independent datasets where available. Second, evidence from observational studies using mouse models supports the role of tocilizumab in treating IS [52-53]. However, this has not been confirmed using RCTs, although RCT evidence found no increased risk of adverse cardiovascular events among RA patients treated with tocilizumab compared with those receiving other biological disease-modifying antirheumatic drugs [54-56].

\section{Conclusions}

Our comprehensive analysis using large-scale GWAS datasets, eQTLs datasets, gene expression datasets, and IS case-control gene expression datasets demonstrated that: (1) the rs7529229 variant minor allele significantly alleviated the risk of IS; (2) the rs7529229 variant minor allele significantly reduced the expression of $I L-6 R$ in relevant tissues or organs especially blood vessels and whole blood; 
(3) IL-6R was mainly expressed in skeletal muscle and whole blood; and (4) IL-6R expression was significantly decreased in the whole blood of healthy controls compared with IS patients. Importantly, the biological senses in the above findings are convergent. Hence, $I L-6 R$ may be a potential therapeutic target for IS, and blocking IL- 6 signaling by means such as tocilizumab might be an effective treatment for IS or method of lowering its risk. This warrants further testing in suitably powered RCTs.

\section{Abbreviations}

IS, ischemic stroke; IL-6, Interleukin-6; IL-6R, IL-6 receptor; sIL-6R, soluble IL-6R; gp130, glycoprotein 130; CVD, cardiovascular diseases; CAD, coronary artery disease; RA, rheumatoid arthritis; CRP, C-reactive protein; GWAS, genome-wide association study; eQTLs, expression quantitative trait loci; MR, Mendelian randomization; RCTs, randomized controlled trials; NSTEMI, non-ST elevation myocardial infarction; LAS, large artery atherosclerotic stroke; CES, cardioembolic stroke; SVS, small vessel stroke; MVP, Million Veteran Program; CARDIoGRAMplusC4D consortium, Coronary ARtery DIsease Genome wide Replication and Meta-analysis (CARD loGRAM) plus The Coronary Artery Disease (C4D) Genetics; Braineac, Brain eQTL Almanac database; GTEx, Genotype-Tissue Expression; TPM, transcripts per million; PEER, Probabilistic Estimation of Expression Residuals; OR, odds ratio; PheWAS, phenome-wide association study;

\section{Declarations}

\section{Ethics approval and consent to participate}

This article includes data from human participants collected by several previous studies. All participants gave their informed consent for participation in all original studies, as described in the Methods. Because our study is based on publicly available large-scale datasets and not individual-level data, ethical approval was not sought.

\section{Consent for publication}

Not applicable.

\section{Availability of data and materials}

All relevant data are within the paper. The authors confirm that all data underlying the findings are either fully available without restriction through consortia websites, or may be made available from consortia upon request. The MEGASTROKE IS GWAS dataset is available from:

http://www.megastroke.org/index.html; the UK Biobank IS GWAS dataset is from: http://pheweb.sph.umich.edu/SAIGE-UKB/; the MVP IS GWAS dataset is from [19] (eTable 1); the RA GWAS dataset is from: http://plaza.umin.ac.jp/ yokada/datasource/software.htm; and the CAD GWAS dataset is from: http://www.cardiogramplusc4d.org/data-downloads/. 


\section{Competing interests}

The authors declare no competing interests.

\section{Funding}

This work was supported by funding from the National Natural Science Foundation of China (Grant No. 82071212, and 81901181), and the Mathematical Tianyuan Fund of the National Natural Science Foundation of China (Grant No. 12026414). This work was also partially supported by funding from the Science and Technology Beijing One Hundred Leading Talent Training Project (Z141107001514006), the Beijing Municipal Administration of Hospitals' Mission Plan (SML20150802).

\section{Authors' contributions}

GYL, XMJ, and WBZ conceived and initiated the project. ZHH and GYL analyzed the data. ZHH, WBZ, and GYL wrote the first draft of the manuscript. All authors contributed to the interpretation of the results and critical revision of the manuscript for important intellectual content and approved the final version of the manuscript.

\section{Acknowledgments}

Prof. Guiyou Liu is a member of the International Stroke Genetics Consortium (ISGC). We thank the ISGC, MEGASTROKE consortium, and METASTROKE consortium for the stroke GWAS datasets. We also thank the UK Brain Expression Consortium (UKBEC), Genotype-Tissue Expression (GTEx), eQTLGen Consortium, and O'Connell et al. for the eQTLs datasets and gene expression dataset. We thank Sarah Williams, PhD, from Liwen Bianji, Edanz Editing China (www.liwenbianji.cn/ac), for editing the English text of a draft of this manuscript.

\section{References}

1. Parisinos CA, Serghiou S, Katsoulis M, George MJ, Patel RS, Hemingway H, Hingorani AD: Variation in Interleukin 6 Receptor Gene Associates With Risk of Crohn's Disease and Ulcerative Colitis.Gastroenterology 2018, 155:303-306 e302.

2. Lambertsen KL, Finsen B, Clausen BH: Post-stroke inflammation-target or tool for therapy?Acta Neuropathol 2019, 137:693-714.

3. Kang S, Tanaka T, Narazaki M, Kishimoto T: Targeting Interleukin-6 Signaling in Clinic./mmunity 2019, 50:1007-1023.

4. Jones $\mathrm{SA}$, Jenkins $\mathrm{BJ}$ : Recent insights into targeting the IL-6 cytokine family in inflammatory diseases and cancer.Nat Rev Immunol 2018, 18:773-789.

5. Garbers C, Heink S, Korn T, Rose-John S: Interleukin-6: designing specific therapeutics for a complex cytokine.Nat Rev Drug Discov 2018, 17:395-412. 
6. Esenwa CC, Elkind MS: Inflammatory risk factors, biomarkers and associated therapy in ischaemic stroke.Nat Rev Neuro/ 2016, 12:594-604.

7. Suzuki S, Tanaka K, Suzuki N: Ambivalent aspects of interleukin-6 in cerebral ischemia: inflammatory versus neurotrophic aspects.J Cereb Blood Flow Metab 2009, 29:464-479.

8. Jenny NS, Callas PW, Judd SE, McClure LA, Kissela B, Zakai NA, Cushman M: Inflammatory cytokines and ischemic stroke risk: The REGARDS cohort.Neurology 2019, 92:e2375-e2384.

9. Swerdlow DI, Holmes MV, Kuchenbaecker KB, Engmann JE, Shah T, Sofat R, Guo Y, Chung C, Peasey A, Pfister R, et al: The interleukin- 6 receptor as a target for prevention of coronary heart disease: a mendelian randomisation analysis.Lancet 2012, 379:1214-1224.

10. Ziegler L, Gajulapuri A, Frumento P, Bonomi A, Wallen H, de Faire U, Rose-John S, Gigante B: Interleukin 6 trans-signalling and risk of future cardiovascular events. Cardiovasc Res 2019, 115:213221.

11. Rosa M, Chignon A, Li Z, Boulanger MC, Arsenault BJ, Bosse Y, Theriault S, Mathieu P: A Mendelian randomization study of IL6 signaling in cardiovascular diseases, immune-related disorders and longevity.NPJ Genom Med 2019, 4:23.

12. Georgakis MK, Malik R, Gill D, Franceschini N, Sudlow CLM, Dichgans M: Interleukin-6 Signaling Effects on Ischemic Stroke and Other Cardiovascular Outcomes: A Mendelian Randomization Study.Circ Genom Precis Med 2020, 13:e02872.

13. Smolen JS, Beaulieu A, Rubbert-Roth A, Ramos-Remus C, Rovensky J, Alecock E, Woodworth T, Alten R: Effect of interleukin-6 receptor inhibition with tocilizumab in patients with rheumatoid arthritis (OPTION study): a double-blind, placebo-controlled, randomised trial.Lancet 2008, 371:987-997.

14. Genovese MC, McKay JD, Nasonov EL, Mysler EF, da Silva NA, Alecock E, Woodworth T, Gomez-Reino $\mathrm{JJ}$ : Interleukin-6 receptor inhibition with tocilizumab reduces disease activity in rheumatoid arthritis with inadequate response to disease-modifying antirheumatic drugs: the tocilizumab in combination with traditional disease-modifying antirheumatic drug therapy study.Arthritis Rheum 2008, 58:29682980.

15. Kremer JM, Blanco R, Brzosko M, Burgos-Vargas R, Halland AM, Vernon E, Ambs P, Fleischmann R: Tocilizumab inhibits structural joint damage in rheumatoid arthritis patients with inadequate responses to methotrexate: results from the double-blind treatment phase of a randomized placebocontrolled trial of tocilizumab safety and prevention of structural joint damage at one year.Arthritis Rheum 2011, 63:609-621.

16. Nishimoto N, Terao K, Mima T, Nakahara H, Takagi N, Kakehi T: Mechanisms and pathologic significances in increase in serum interleukin- 6 (IL-6) and soluble IL- 6 receptor after administration of an anti-IL-6 receptor antibody, tocilizumab, in patients with rheumatoid arthritis and Castleman disease.Blood 2008, 112:3959-3964.

17. DeMizio DJ, Geraldino-Pardilla LB: Autoimmunity and Inflammation Link to Cardiovascular Disease Risk in Rheumatoid Arthritis.Rheumatol Ther 2020, 7:19-33. 
18. Sarwar N, Butterworth AS, Freitag DF, Gregson J, Willeit P, Gorman DN, Gao P, Saleheen D, Rendon A, Nelson CP, et al: Interleukin-6 receptor pathways in coronary heart disease: a collaborative metaanalysis of 82 studies.Lancet 2012, 379:1205-1213.

19. Cai T, Zhang Y, Ho YL, Link N, Sun J, Huang J, Cai TA, Damrauer S, Ahuja Y, Honerlaw J, et al: Association of Interleukin 6 Receptor Variant With Cardiovascular Disease Effects of Interleukin 6 Receptor Blocking Therapy: A Phenome-Wide Association Study.JAMA Cardiol 2018, 3:849-857.

20. Ward LD, Kellis M: HaploReg v4: systematic mining of putative causal variants, cell types, regulators and target genes for human complex traits and disease.Nucleic Acids Res 2016, 44:D877-881.

21. Liu G, Zhang H, Liu B, Wang T, Han Z, Ji X: rs4147929 variant minor allele increases ABCA7 gene expression and ABCA7 shows increased gene expression in Alzheimer's disease patients compared with controls.Acta Neuropathol 2020, 139:937-940.

22. Liu G, Zhang H, Liu B, Ji X: Rs2293871 regulates HTRA1 expression and affects cerebral small vessel stroke and Alzheimer's disease.Brain 2019, 142:e61.

23. Liu G, Hu Y, Han Z, Jin S, Jiang Q: Genetic variant rs17185536 regulates SIM1 gene expression in human brain hypothalamus. Proc Natl Acad Sci U S A 2019, 116:3347-3348.

24. Liu G, Jin S, Hu Y, Jiang Q: Disease status affects the association between rs4813620 and the expression of Alzheimer's disease susceptibility gene TRIB3.Proc Natl Acad Sci U S A 2018, 115:E10519-E10520.

25. Hu Y, Jin S, Cheng L, Liu G, Jiang Q: Autoimmune disease variants regulate GSDMB gene expression in human immune cells and whole blood.Proc Natl Acad Sci U S A 2017, 114:E7860-E7862.

26. Liu G, Hu Y, Jiang Q: Population Difference and Disease Status Affect the Association Between Genetic Variants and Gene Expression.Gastroenterology 2019, 157:894-896.

27. Paige E, Clement M, Lareyre F, Sweeting M, Raffort J, Grenier C, Finigan A, Harrison J, Peters JE, Sun BB, et al: Interleukin-6 Receptor Signaling and Abdominal Aortic Aneurysm Growth Rates.Circ Genom Precis Med 2019, 12:e002413.

28. Usategui-Martin R, Lendinez-Tortajada V, Perez-Castrillon JL, Briongos-Figuero L, Abadia-Otero J, Martin-Vallejo J, Lara-Hernandez F, Chaves FJ, Garcia-Garcia AB, Martin-Escudero JC:

Polymorphisms in genes involved in inflammation, the NF-kB pathway and the renin-angiotensinaldosterone system are associated with the risk of osteoporotic fracture. The Hortega Follow-up Study.Bone 2020, 138:115477.

29. Wosiski-Kuhn M, Robinson M, Strupe J, Arounleut P, Martin M, Caress J, Cartwright M, Bowser R, Cudkowicz M, Langefeld C, et al: IL6 receptor(358)Ala variant and trans-signaling are disease modifiers in amyotrophic lateral sclerosis.Neurol Neuroimmunol Neuroinflamm 2019, 6:e631.

30. Hansen PR, Nelveg-Kristensen KE, Rasmussen HB, Torp-Pedersen C, Kober L, Nielsen CH, Enevold C: Prognostic role of genetic polymorphisms of the interleukin-6 signaling pathway in patients with severe heart failure. Pharmacogenomics J 2019, 19:428-437.

31. Key KV, Mudd-Martin G, Moser DK, Rayens MK, Morford LA: Inflammatory Genotype Moderates the Association Between Anxiety and Systemic Inflammation in Adults at Risk for Cardiovascular 
Disease.J Cardiovasc Nurs 2020.

32. Ferreira MA, Matheson MC, Duffy DL, Marks GB, Hui J, Le Souef P, Danoy P, Baltic S, Nyholt DR, Jenkins $\mathrm{M}$, et al: Identification of IL6R and chromosome 11q13.5 as risk loci for asthma.Lancet 2011, 378:1006-1014.

33. Naitza S, Porcu E, Steri M, Taub DD, Mulas A, Xiao X, Strait J, Dei M, Lai S, Busonero F, et al: A genome-wide association scan on the levels of markers of inflammation in Sardinians reveals associations that underpin its complex regulation.PLoS Genet 2012, 8:e1002480.

34. Kleveland O, Kunszt G, Bratlie M, Ueland T, Broch K, Holte E, Michelsen AE, Bendz B, Amundsen BH, Espevik T, et al: Effect of a single dose of the interleukin-6 receptor antagonist tocilizumab on inflammation and troponin T release in patients with non-ST-elevation myocardial infarction: a double-blind, randomized, placebo-controlled phase 2 trial.Eur Heart J 2016, 37:2406-2413.

35. Helseth R, Kleveland O, Ueland T, Wiseth R, Damaas JK, Broch K, Michelsen AE, Bendz B, Gullestad L, Aukrust $P$, Seljeflot I: Effect of a single dose of the interleukin-6 receptor antagonist tocilizumab on markers of neutrophil extracellular traps in patients with non-ST elevation myocardial infarction.JACC 2020, 75:154.

36. Gill D, Georgakis MK, Koskeridis F, Jiang L, Feng Q, Wei WQ, Theodoratou E, Elliott P, Denny JC, Malik $\mathrm{R}$, et al: Use of Genetic Variants Related to Antihypertensive Drugs to Inform on Efficacy and Side Effects.Circulation 2019, 140:270-279.

37. Chong M, Sjaarda J, Pigeyre M, Mohammadi-Shemirani P, Lali R, Shoamanesh A, Gerstein HC, Pare G: Novel Drug Targets for Ischemic Stroke Identified Through Mendelian Randomization Analysis of the Blood Proteome.Circulation 2019, 140:819-830.

38. Malik R, Chauhan G, Traylor M, Sargurupremraj M, Okada Y, Mishra A, Rutten-Jacobs L, Giese AK, van der Laan SW, Gretarsdottir S, et al: Multiancestry genome-wide association study of 520,000 subjects identifies 32 loci associated with stroke and stroke subtypes.Nat Genet 2018, 50:524-537.

39. Bycroft C, Freeman C, Petkova D, Band G, Elliott LT, Sharp K, Motyer A, Vukcevic D, Delaneau O, O'Connell J, et al: The UK Biobank resource with deep phenotyping and genomic data.Nature 2018, 562:203-209.

40. Ramasamy A, Trabzuni D, Guelfi S, Varghese V, Smith C, Walker R, De T, Coin L, de Silva R, Cookson $\mathrm{MR}$, et al: Genetic variability in the regulation of gene expression in ten regions of the human brain.Nat Neurosci 2014, 17:1418-1428.

41. The Genotype-Tissue Expression (GTEx) project.Nat Genet 2013, 45:580-585.

42. Võsa. U, Claringbould. A, Westra. H-J, Bonder. MJ, Deelen. P, Zeng. B, Kirsten. H, Saha. A, Kreuzhuber. $\mathrm{R}$, Kasela. S, Pervjakova. N: Unraveling the polygenic architecture of complex traits using blood eQTL meta analysis.bioRxiv 2018.

43. Battle A, Brown CD, Engelhardt BE, Montgomery SB: Genetic effects on gene expression across human tissues. Nature 2017, 550:204-213.

44. O'Connell GC, Petrone AB, Treadway MB, Tennant CS, Lucke-Wold N, Chantler PD, Barr TL: Machinelearning approach identifies a pattern of gene expression in peripheral blood that can accurately 
detect ischaemic stroke.NPJ Genom Med 2016, 1:16038.

45. Okada Y, Wu D, Trynka G, Raj T, Terao C, Ikari K, Kochi Y, Ohmura K, Suzuki A, Yoshida S, et al: Genetics of meumatoid arthritis contributes to biology and drug discovery.Nature 2014, 506:376-381.

46. Nikpay M, Goel A, Won HH, Hall LM, Willenborg C, Kanoni S, Saleheen D, Kyriakou T, Nelson CP, Hopewell JC, et al: A comprehensive 1,000 Genomes-based genome-wide association meta-analysis of coronary artery disease.Nat Genet 2015, 47:1121-1130.

47. A genome-wide association study in Europeans and South Asians identifies five new loci for coronary artery disease.Nat Genet 2011, 43:339-344.

48. Liu G, Xu Y, Jiang Y, Zhang L, Feng R, Jiang Q: PICALM rs3851179 Variant Confers Susceptibility to Alzheimer's Disease in Chinese Population.Mol Neurobio/ 2017, 54:3131-3136.

49. Barrett T, Wilhite SE, Ledoux P, Evangelista C, Kim IF, Tomashevsky M, Marshall KA, Phillippy KH, Sherman PM, Holko M, et al: NCBI GEO: archive for functional genomics data sets-update.Nucleic Acids Research 2013, 41:D991-D995.

50. Appelros P, Stegmayr B, Terent A: Sex differences in stroke epidemiology: a systematic review.Stroke 2009, 40:1082-1090.

51. Hunter CA, Jones SA: IL-6 as a keystone cytokine in health and disease.Nat Immuno/ 2015, 16:448457.

52. Hudobenko J, Verma R, McCullough L: Interleukin-6 Receptor Inhibition with Tocilizumab Ameliorates Ischemic Stroke Damage in Mice.Stroke 2017, 48:ATP270.

53. Hudobenko J, Chauhan A, McCullough L: Inhibition of Interleukin-6 Signaling With Tocilizumab Ameliorates Ischemic Stroke Damage in Aged Male and Female Mice.Stroke 2018, 49:A28.

54. Giles JT, Sattar N, Gabriel S, Ridker PM, Gay S, Warne C, Musselman D, Brockwell L, Shittu E, Klearman M, Fleming TR: Cardiovascular Safety of Tocilizumab Versus Etanercept in Rheumatoid Arthritis: A Randomized Controlled Trial.Arthritis Rheumatol 2020, 72:31-40.

55. Castagne B, Viprey M, Martin J, Schott AM, Cucherat M, Soubrier M: Cardiovascular safety of tocilizumab: A systematic review and network meta-analysis.PLoS One 2019, 14:e220178.

56. Sepriano A, Kerschbaumer A, Smolen JS, van der Heijde D, Dougados M, van Vollenhoven R, Mclnnes IB, Bijlsma JW, Burmester GR, de Wit M, et al: Safety of synthetic and biological DMARDs: a systematic literature review informing the 2019 update of the EULAR recommendations for the management of rheumatoid arthritis.Ann Rheum Dis 2020, 79:760-770.

\section{Figures}




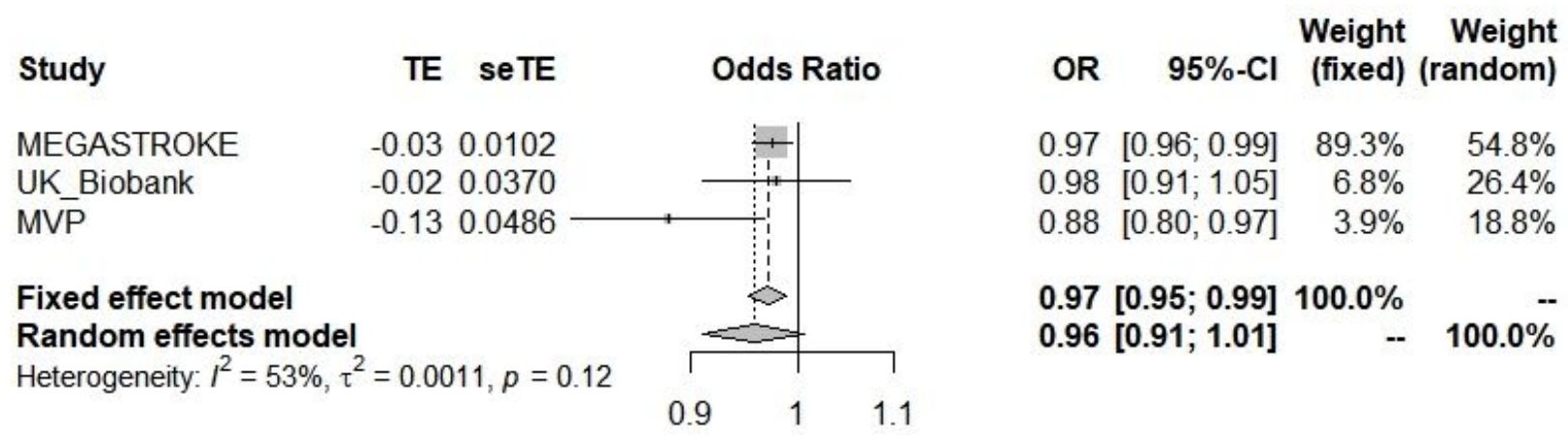

\section{Figure 1}

Forest plot for the meta-analysis of rs7529229 variant in MEGASTROKE, UK Biobank, and MVP M-H, Mantel-Haenszel; Fixed, fixed effect model; Cl, confidence interval;

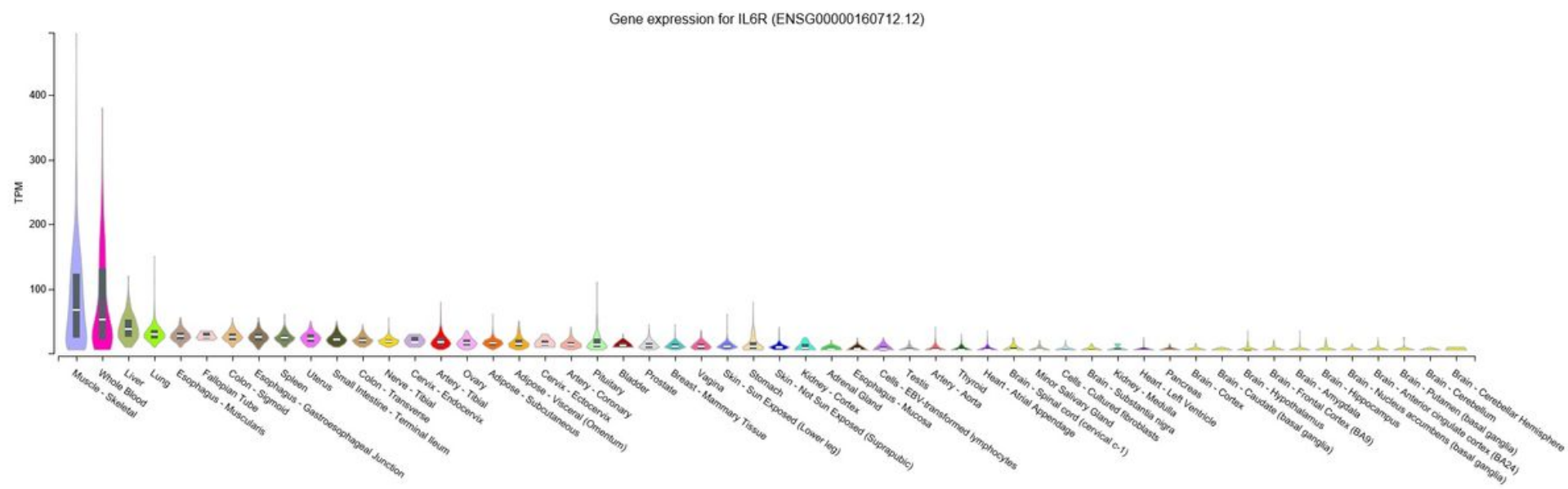

\section{Figure 2}

Box plots for the expression of IL-6R gene in different tissues in GTEx The gene expression values are shown in transcripts per million (TPM). The gene expression level was quantified byTPM based on the GENCODE 26 annotation, collapsed to a single transcript model for each gene using a custom isoform collapsing procedure [43]. 